\title{
Klaus-Dieter Die soziale Verantwortung Altmeppen des Journalismus
}

\section{Die „Bananenuniversität" und das Problem sozialer Verantwortung'}

Es ist keine schöne Charakterisierung, die die Katholische Universität Eichstätt-Ingolstadt (KU) jüngst erhalten hat. Von einer „Bananenuniversität" schrieb die Presse (vgl. „Donaukurier“ vom 21.5.2008). Der Urheber dieses gruselig-schönen Wortes, ein Mitglied der KU, befand sich in Afrika, als er von einer Zeitung interviewt wurde zur Krise der KU. Er hat - unter Anspielung auf seinen Aufenthaltsort - darauf hingewiesen, dass es sich bei der KU gerade nicht um eine „Bananenuniversität" handele. Das ist von der Zeitung auch korrekt wiedergegeben worden. In der zweiten Zeitung allerdings, Journalisten schreiben auch schon gern mal von Kollegen $a b$, ist dann nur noch die "Bananenuniversität" übrig geblieben.

Diese Charakterisierung ist Teil einer Berichterstattung, die - wie in vielen anderen Fällen - den Beteiligten häufig genug nicht gefällt. Die Berichterstattung über nachrichtenwerte Ereignisse wie an der KU (die Nicht-Ernennung eines gewählten Präsidenten, der Rücktritt mehrerer Angehöriger der Hochschulleitung) gehört aber zu den Pflichten der Journalisten. Sie hören berufsmäßig besonders deutlich hin, sie schärfen ihre Federn und berichten besonders intensiv. Häufig sprechen die Beteiligten und Betroffenen dann von einer Medienkrise, eben weil die Berichterstattung nicht so ist, wie die Beteiligten die Geschehnisse erleben. Vergessen wird dabei leicht, dass zwischen der Krise und der Kommunikationskrise erhebliche Unterschiede bestehen. Gewiss kann die öffentliche Berichterstattung eine Krise verschärfen, die Berichterstattung ist aber nicht die Krise.

Zwei Erklärungen bieten sich an, um das Gerede von der Medienkrise zu erklären. Entweder versuchen die Betroffenen und Beteiligten, jene also, die undifferenzierte Medienschelte betreiben, von der eigentlichen Krise abzulenken. Es ist eben leichter, die Medien zum Sündenbock zu machen, als die Krise zu bewältigen. Ebenso häufig

1 Der Beitrag ist die leicht veränderte Fassung der Antrittsvorlesung an der Katholischen Universität Eichstätt-Ingolstadt am 17. Juli 2008. 
aber dürfte schlichte Unkenntnis über die Mechanismen und Strukturen der Berichterstattung der Grund sein, dass Betroffene und Beteiligte die Berichterstattung kritisieren.

Der Grund dafür ist recht simpel: Im Sinne der Konstruktion von Wirklichkeit nimmt jeder Beteiligte und Betroffene die Ereignisse unterschiedlich wahr. Das gilt auch für die Journalisten. Damit die Berichterstattung aber nun nicht beliebig wird, gibt es professionelle Kriterien, die bei der Berichterstattung anzulegen sind. Was ausgewählt wird (vorzugsweise Aktuelles), wird über Nachrichtenfaktoren entschieden, und wie es produziert wird, entscheidet sich über die redaktionellen Strukturen.

Nun argumentieren Betroffene wie die Mitglieder der KU, dass endlich wieder positive Nachrichten ïber die KU erscheinen müssten. Und dafür, bitte schön, solle doch die Pressestelle sorgen, das sei ja ihre Aufgabe. Daran ist zweierlei auffällig:

1. Das Spiel, das in solchen Fällen beginnt, heißt Schwarzer Peter. Verlierer ist immer der Pressesprecher. Er hat die schlechtesten Karten. Und die sind leider auch noch gezinkt, denn was veröffentlicht werden soll, bestimmen selten die PR-Spezialisten, meistens dagegen die Entscheider. Die müssen sich auch an die Spielregeln der Mediengesellschaft halten. Dazu gehört: Only bad news is good news. Um diese Spielregeln umzudrehen, um auch positive Nachrichten in die Medien zu bekommen, braucht es mehr als einen Pressesprecher. Dazu braucht es ein Team an professionellen Kommunikatoren, und dazu braucht es den Willen aller Mitglieder betroffener Organisationen, Ereignisse mit positiven Inhalten zu produzieren. Glaubwürdige PR ist nie besser als das Objekt, das sie kommuniziert.

2. Es gehört zu den eindeutigen Ergebnissen der Kommunikationsund Medienwissenschaften, dass der Journalismus auch dadurch unter Druck kommt, dass immer mehr Pressemitteilungen die $\mathrm{Re}$ daktionen erreichen. Nicht nur die $\mathrm{KU}$, auch das Landratsamt, der $\mathrm{Ca}$ ritasverband, die Fußballvereine, sie alle möchten ihre Mitteilungen veröffentlicht sehen. Vornehmste Aufgabe des Journalisten ist es, aus dieser Flut das auszuwählen, was die größte Aktualität, die größte Nähe zu den Zielgruppen und die höchste Relevanz hat. Da fällt einiges unter den Tisch statt ins Blatt zu kommen. Denn leider stimmt nicht, was gem kolportiert wird: dass gerade immer soviel passiert, wie in eine Zeitung passt. Es passiert viel mehr, und die, die im $\mathrm{Pa}$ pierkorb landen, sind dann alle frustriert. Die Ironie ist, dass genau die Forderung, nun müsse die Pressestelle doch endlich publizieren, zu exakt der Informationsflut führt, die den Journalismus unter Druck setzt (vgl. Altmeppen/Röttger/Bentele 2004). 
Frustriert sind auch jene, deren Aussagen in den Medien zitiert werden, nur leider nicht so, wie es ausgesprochen wurde. Auch daraus entsteht die weithin vernehmbare Medienschelte: Diese Journalisten, nie berichten sie die Wahrheit, immer verdrehen sie die Fakten!

Dies mag manchmal der Fall sein - auch unter den Journalisten finden sich etliche, die Politik durch Berichterstattung machen. Dies ist jedoch die Ausnahme. Weit mehr verantwortlich dafür, dass die Berichterstattung nicht der Realität entspricht, sind die Strukturen des Journalismus. Strukturen, das sind all jene Faktoren, die jeden Journalisten bei seiner Berufsausübung beeinflussen (vgl. Altmeppen 2006). Gemeint sind also zum Beispiel die Regeln der Auswahl von Nachrichten; die Zeit, die nötig ist, um zu recherchieren und zu schreiben; der Platz, der zur Verfügung steht für den Beitrag.

\section{Die soziale Verantwortung des Journalismus}

Das führt mich zu meiner ersten These über die soziale Verantwortung des Journalismus. Diese Verantwortung liegt nicht darin, sich etwa Gedanken darïber machen zu müssen, welche Wirkung der Begriff "Bananenuniversität" hat. Journalistische Berichterstattung ist immer das Ergebnis sozialen Handelns, das Ergebnis der Arbeit in der Redaktion, und bei sozialem Handeln entstehen immer unintendierte - und damit unabschätzbare - Folgen und Wirkungen.

Dies bedeutet jedoch keinen Freispruch für den Journalismus, denn die soziale Verantwortung des Journalisten besteht darin, eine professionelle Arbeit abzuliefern. Das schnöde Abschreiben des schönen Begriffs wegen („Bananenuniversität“) ist unprofessionell. Professionell handelt der Journalist, wenn er Fakten recherchiert, wenn er auch die andere Seite hört, wenn er sich ein Bild der Geschehnisse macht, das den Ansprïchen von Wahrhaftigkeit und Objektivität zumindest nacheifert. Professionell handelt der Journalismus, wenn ein Gegenlesen stattfindet, wenn also nach dem Vier-Augen-Prinzip eine organisatorisch etablierte Kontrolle stattfindet.

1. These: Der Joumalismus ist der Produzent der medialen Inhalte, und für diesen Produktionsprozess ist er verantwortlich. Nur mit dieser sozialen Verantwortung kann der Joumalismus seine Leistungen für die Gesellschaft legitimieren.

Die soziale Verantwortung des Journalismus, die auf ethischen und professionellen Grundlagen fußt, hat somit drei Ebenen: eine indivi- 
duelle, dort, wo jeder Journalist für sein Handeln soziale Verantwortung übernimmt; eine organisationale Ebene, bei der die journalistische Organisation sich über ihre soziale Verantwortung klar sein muss (in den Redaktionen entscheidet sich, inwieweit ethische Standards eine Rolle spielen); und schließlich eine professionelle Ebene, auf der sich die Profession der Journalisten über soziale Verantwortung verständigen muss.

Nun wird aller sozialen Verantwortung zum Trotz der Begriff der „Bananenuniversität" publik. Da hat dann also wohl, so könnte man schlussfolgern, die soziale Verantwortung nicht gegriffen. Also wird mit Medienschelte draufgehauen. Die Journalisten, sowieso ja eine abgehobene Elite, sind nicht kontrollierbar, was besonders verwerflich ist, weil sie ja eigene politische Ziele verfolgen oder Meinungsjournalismus betreiben.

Aber Vorsicht, das ist trügerisch! Leider, und damit bin ich bei den Zwängen, tragen die Medienunternehmen einiges dazu bei, die Spielräume, die ein sozial verantwortlicher Joumalismus benötigt, einzuengen.

\section{Die soziale Verantwortung der Medien}

Nun wird zu hören sein: Ja klar, Medien und Journalismus, das ist doch, wenn nicht dasselbe, so doch zumindest das Gleiche. Nein, so meine zweite These, weder das eine noch das andere! Man muss für diese Vorstellung allerdings den traditionellen Weg des In-EinsDenkens von Journalismus und Medien verlassen und sich auf neue Spuren begeben.

\section{These: Journalismus und Medien sind nicht das Gleiche.}

Noch einmal: Journalismus produziert Inhalte, findet also Themen, recherchiert $\mathrm{zu}$ diesen Themen, bereitet die Themen auf für Print, Hörfunk, Fernsehen, Online. Journalismus kann aber nicht distribuieren. Dazu benötigt der Journalismus die Medien, denen er die Inhalte „verkauft“. Die Kernkompetenz der Medien ist es, Inhalte (Nachrichten, Unterhaltung, Werbung) zu distribuieren, denn die Distributionstechniken gehören den Medienorganisationen. Sie sind die Besitzer und Kapitaleigner für Druckstraßen, sie verfügen über die Senderechte, sie haben die Webseiten mitsamt Server und Datenleitung gekauft. Man kann folglich das Verhältnis von Journalismus und Medien als das von Verkäufer und Käufer beschreiben (vgl. Altmeppen 2006). 


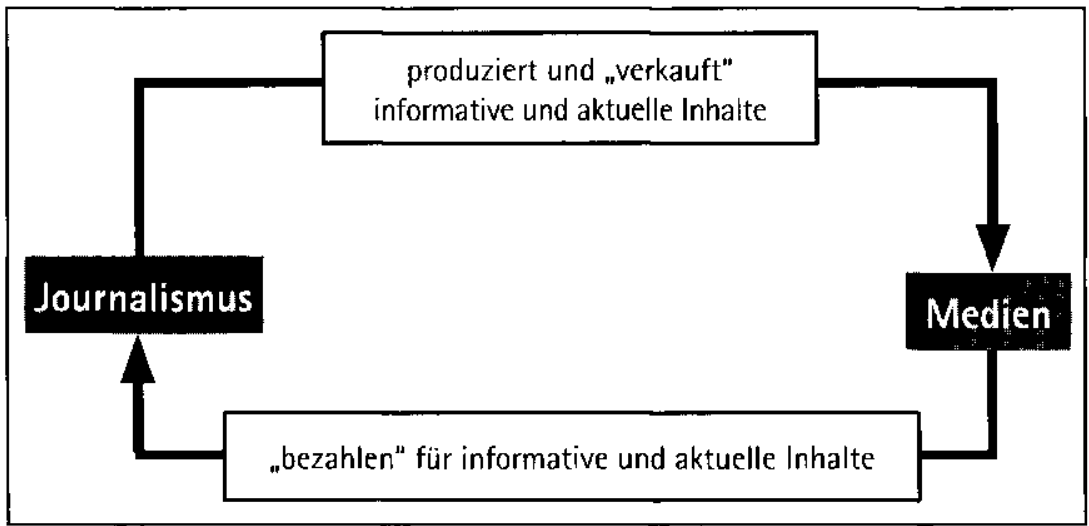

Das Verhältnis von Journalismus und Medien

Kaufen und verkaufen ist in einem symbolischen Sinn zu verstehen. Der Journalismus benötigt für seine produzierten Inhalte einen Abnehmer, der die Inhalte an die Zielgruppen verteilt. Dieser Abnehmer sind die Medien. Sie benötigen Inhalte für ihr Geschäft der Distribution. In diesem Tauschgeschäft fließt Geld nur indirekt. Die Medien bezahlen nicht unmittelbar für die journalistischen Leistungen; sie statten den Journalismus mit den notwendigen Ressourcen aus: mit Gehältern für die Journalisten, mit Räumen für die Arbeit, mit Technik für die Produktion. Aufgrund der engen Beziehungen zwischen den Medienorganisationen und den journalistischen Organisationen kann deren Verhältnis als das einer Ko-Orientierung bezeichnet werden. Erst aufgrund dieser Ko-Orientierung kommt der mediale Kommunikationsprozess der Information (durch den Journalismus), der Mitteilung (durch die Medienorganisationen) und des Verstehens (durch die Rezipienten) zustande.

Diese Ansicht ist gar nicht so neu. Schon frühe Kommunikationsmodelle - von Lasswell beispielsweise oder von Maletzke - konstatieren die Unterscheidungen von Journalist und Medium. Bei Lasswell ist der Kommunikator durch das "who" bezeichnet, die Medien durch „in which channel“. Bei Maletzke gehen die Differenzierungen noch weiter, etwa wenn er bestimmte Zwänge zwischen Kommunikator und Medium analysiert (vgl. Burkart/Hömberg 2007, S. 260). Allerdings waren die Modelle sozusagen Reintypen, die den Kommunikationsprozess beschreiben, ohne dass damit über Ursachen oder Folgen weitere Aussagen gemacht würden. Eine dieser Folgen, Journalismus und Medien $z \mathfrak{u}$ trennen, liegt darin, dass die Frage nach sozialer Verantwortung ebenso differenziert beantwortet werden muss. Folgt man dieser Unterscheidung, führt uns dies zur dritten These. 
These 3: Die soziale Verantwortung der Medien liegt darin, dem Journalismus die notwendigen Ressourcen für die Berichterstattung zur Verfügung zu stellen.

Wenn Journalismus und Medien nicht das Gleiche sind, sondern sich in ihren Funktionen und Leistungen unterscheiden, so unterscheiden sich auch die Grundlagen und Maßstäbe der sozialen Verantwortung. Damit tragen die Medienunternehmen nicht mehr die Verantwortung für die Berichterstattung, die obliegt dem Joumalismus. Die soziale Verantwortung der Medien liegt darin, dem Journalismus die Ressourcen zur Verfügung zu stellen, die er für eine Berichterstattung benötigt. Wir haben es also mit einer gesplitteten Verantwortung zu tun. Das hat erhebliche Folgen. So können die Ressourcen, die die Medien dem Journalismus zur Verfügung stellen, quantitativ bewertet werden. Ob Redaktionen Mitarbeiter einstellen oder entlassen, ob den Journalisten zusätzliche Aufgaben wie die Produktion von Onlineausgaben aufgebürdet werden, sind unter Ressourcengesichtspunkten messbare Parameter, die Aussagen auch darüber erlauben, ob eine qualitativ hochwertige Berichterstattung möglich ist oder nicht.

\section{Krisensymptome und soziale Verantwortung}

Wirft man unter diesem Gesichtspunkt einen kurzen Blick auf die derzeitigen Verhältnisse im Medienbereich, so zeigt sich, wie sehr das Verantwortungsbewusstsein insbesondere der Medienunternehmen fehlt und wie nötig eine Auseinandersetzung über eine gesplittete soziale Verantwortung ist, was zu meiner nächsten These führt.

These 4: Die derzeitigen Krisensymptome des Journalismus sind wesentlich darauf zurückzuführen, dass die Medienunternehmen ihre soziale Verantwortung nicht ausreichend wahrnehmen.

Unzweifelhaft lassen sich derzeit Krisensymptome des Journalismus beobachten, die, schaut man genauer hin, ihre Ursache deutlich stärker in der fehlenden sozialen Verantwortung der Medien haben als in der des Journalismus. Was in einem schleichenden, aber offensichtlichen Prozess aktuell passiert, ist anscheinend so etwas wie die - deutlich verspätete - Industrialisierung dieses Berufes. Im Zeichen technologischer Entwicklung (Digitalisierung) und unter den Bedingungen einer wachsenden Konzentration des Kapitals entstehen zunehmend prekäre Arbeitsverhältnisse im Journalismus. Der Beruf wird entzaubert und entmythologisiert. 
Der Journalist, ein kreativer, künstlerischer, freier, Kultur produzierender Mensch: Machen wir uns - und vor allem denjenigen, die diesen Beruf ergreifen wollen - nichts vor, diese Zeiten scheinen vorbei zu sein. So wenig wie der Schlosser im Maschinenbau oder der Karosseriebauer im Autowerk entscheidet der Journalist frei und kreativ darüber, was er tut und was er tun kann. Das tun die Manager für ihn, die in der Medienorganisation sitzen und Geld dafür bekommen, dass sie die Rendite erhöhen oder hoch halten, Geld, das für die Berichterstattung fehlt. Anders kann man die derzeitigen Entwicklungen bei Zeitungsverlagen jedenfalls kaum kennzeichnen. Ein paar Beispiele:

- Zunehmend gibt es Zeitungen "oT", das heißt ohne Tarifvertrag. Die Verlage sind aus den Tarifpartnerschaften ausgestiegen, bei Neueinstellungen wird unter Tarif bezahlt.

- Mit Abfindungsmodellen werden Redakteure, die freiwillig ihre Festanstellung aufgeben, mit einer Abfindungssumme bezahlt und als Freie für 2000 Euro Monatsbruttolohn wieder eingestellt.

- Auch die Beschäftigung von Journalisten als Leiharbeitnehmer nimmt zu. Leihjournalisten werden unter den Redakteurstarifen bezahlt, zum Teil gründen die Verlage für diese Konstellationen eigene Tochter-Leiharbeitsfirmen.

- Beiträge zum Presseversorgungswerk werden nicht bezahlt; damit wird die Altersversorgung der Journalisten verschlechtert.

- Bei der „Münsterschen Zeitung“ wurden im Januar 2007 von einem Tag auf den anderen 17 Lokalredakteure gekündigt, deren Arbeit von einer - zuvor neu gegründeten - Firma mit neuen Redakteuren zu untertariflichen Konditionen übernommen wurde.

Prekäre Zeiten, prekäre Arbeitsverhältnisse, so muss man das wohl nennen. Übrigens sind die Zeitungen nicht die einzigen Medienunternehmen, die permanent Ressourcen für journalistische Leistungen herunterfahren. Der Fernsehsender VOX kommt ohne Nachrichtensprecher aus und hat sein Informationsangebot, ebenso wie Kabel1 oder RTL II, gerade noch soweit erhalten, dass die Sendelizenz als Vollprogramm nicht gefährdet ist. Die großen privaten Sender wie RTL, PR07 und SAT.1 haben ihre Informationserstellung gebündelt. Längst arbeiten für jede Nachrichtensendung nicht mehr eigene Redaktionen. Stattdessen produzieren ausgelagerte Töchter die "News“, deren Personal oft als Feste Freie arbeiten, ohne Vertragssicherheit. Und es wird nicht mehr exklusiv produziert. Die Redaktionen produzieren, als Tochtergesellschaften, für mehrere Sendungen. So werden an vielen Stellen aus den Redaktionsleitern, bislang vor allem Journalisten, Kaufleute, die nicht auf die Ereignishaftigkeit schauen, sondem den Absatz der Produkte bei den Zielgruppen sichern müssen. 
Ein derzeit besonders prägnantes Beispiel für Profit statt Qualität ist die „Berliner Zeitung“, und Josef Depenbrock ist das Gesicht dieser Entwicklungen (vgl. Pimpl 2008). Er ist sozusagen ein doppelter Macher: ein Blattmacher, denn er ist Chefredakteur der "Berliner Zeitung" - und ein Plattmacher, denn er ist gleichzeitig Geschäftsführer dieser Zeitung. Als solcher verantwortet er gerade einen rigorosen Stellenabbau in Verlag und Redaktion. Depenbrock ist davon überzeugt, dass auch 90 (statt 130) Redakteure das Blatt machen können, wohlgemerkt ohne Qualitätsverlust. Depenbrocks Chef ist ein britischer Investor, der gerade verkündet hat, dass seine Objekte (also auch die „Berliner Zeitung“) mindestens 20 Prozent Rendite erbringen müssen. Der Investor steht damit aber nicht allein, denn auch andere Verlage haben kundgetan, dass sie nun endlich wieder in die zweistellige Gewinnzone kommen möchten (Pimpl 2008).

Das alles sind Krisensymptome, Krisensymptome der Medienunternehmen, die auf der Suche nach Leserschaft und Rezipienten also nach Einnahmen - Probleme haben und deshalb die Kosten nach unten treiben.

Ein beliebtes Instrument der Kostendrückerei ist das Personal: weniger Personal, schlechter bezahltes Personal. Und so werden die Ressourcen des Journalismus an seiner empfindlichsten Stelle gekürzt. Personalreduzienung geht grundsätzlich zu Lasten der Qualität. Weniger Personal heißt mehr Berichterstattung durch weniger Leute. Geringer entlohntes Personal heißt, weniger Motivation für die journalistische Arbeit. Was sollen wir an journalistischer Berichterstattung erwarten, wenn die Journalisten wie die Wanderburschen durch die Lande ziehen, um als Leiharbeiter ihr Geld zu verdienen? Was sind ethische Ansprüche an den Journalismus noch wert, wenn die Medienunternehmen allzu deutlich machen, dass sie den Wert der Arbeit, die die Journalisten machen, immer weniger schätzen und honorieren?

Damit kein Missverständnis aufkommt: Medien waren immer erwerbswirtschaftliche Betriebe und selbst die öffentlich-rechtlichen Anstalten sind der Wirtschaftlichkeit verpflichtet. An dieser erwerbswirtschaftlichen Ausrichtung gibt es auch nichts zu kritisieren. Problematisch wird es, wenn die Medienunternehmen wie derzeit das erwerbswirtschaftliche Prinzip in den Vordergrund rïcken und dabei die publizistischen Ziele völlig aus den Augen verlieren; wenn ihnen die Qualität wenig, die Profitsicherung aber enorm viel bedeutet; wenn bislang guiltige Mindeststandards wie tarifvertragliche Bezahlung und dauerhafte Bindung der Journalisten an das Unternehmen weit weniger bedeutsam erscheinen als die kurzfristigen Renditeziele. 
Zusammenfassend kann konstatiert werden, dass erhebliche Unterschiede bestehen zwischen Medien und Journalismus. Das Ziel von Medienunternehmen ist die Profitmaximierung, das Ziel des Journalismus ist die Erkenntnismaximierung. Journalismus ist Dienst am Gemeinwohl, Medien dienen dem Markt. Der Journalismus konkurriert auf dem Markt der Meinungen, die Medien konkurrieren auf dem Absatzmarkt. Es erscheint sinnvoller, diese Unterschiede anzuerken. nen, als dauerhaft weiterhin von Medien und Journalismus als einer unterschiedslosen Entität zu sprechen.

Diese Unterschiede sollten auch bei der Frage nach der jeweiligen Verantwortung beachtet werden. Dann nämlich stellen sich die Verantwortungsfragen nicht nur akzentuierter, sondern auch anders. Das soll im letzten Abschnitt geschehen.

\section{Konsequenzen einer gesplitteten sozialen Verantwortung zwischen Medien und Journalismus}

Um Verantwortung für mediale Berichterstattung adressieren zu können, braucht es Verantwortliche und eine Orientierung für das $\mathrm{Maß}$ an Verantwortung. Wir beobachten ja nicht Verantwortung an sich, sondern die Übernahme oder die Ablehnung von Verantwortung. Niemand wird sich als verantwortungslos bezeichnen. Wenn es um Verantwortungsbewusstsein geht, stimmen alle zu.

Um aber dieses Verantwortungsbewusstsein wirklich beurteilen $\mathrm{zu}$ können, ist zum einen eine Referenz notwendig, ein Orientierungspunkt, an dem gemessen werden kann, ob Verantwortung übernommen wird oder nicht. Zum Zweiten braucht es die Zuschreibung, also die Feststellung, wer denn Verantwortung zu übernehmen hat. Dies sind die Bedingungen sozialer Verantwortung generell und damit auch der sozialen Verantwortung von Journalismus und Medien.

Solange Medien und Journalismus in einen Topf geworfen werden, lassen sich weder die Verantwortlichen noch die Referenzpunkte genau bestimmen. Wer ist für den publizistischen Erfolg oder Misserfolg verantwortlich? Die Redaktion, das Redaktionsmanagement, der einzelne Journalist? Wer ist für den wirtschaftlichen Erfolg oder Misserfolg verantwortlich? Der Verlag, der Programmdirektor, die Geschäftsführung, die Redaktion?

Die Mediengeschichte ist voll von Beispielen, mit denen der Konflikt von journalistischen und ökonomischen Entscheidungskriterien illustriert werden kann. Nur ein Beispiel: Ist das Interesse der Öffentlichkeit am Bild des toten Uwe Barschel in der Badewanne gerechtfertigt, so dass auch mit journalistischer Verantwortung die Veröf- 
fentlichung gerechtfertigt ist? Oder war das ökonomische Interesse des „Stem“ an einer Auflagensteigerung so groß, dass das Bild veröffentlicht wurde?

Otfried Jarren plädiert zur Lösung dieser Frage dafür, dass es kulturell eingebundenes Kapital brauche, damit die ökonomischen nicht die publizistischen Rationalitäten dominieren (vgl. Jarren/Zwicky 2008). Nur wenn die Medieneigner auch den kulturellen Wert der Medien in ihre Investitionsentscheidungen einbeziehen, könnte ein Freiraum für guten Journalismus entstehen. Er bricht damit das Problem von der ökonomischen Seite, der Seite der Medieneigner auf, deren Investitionen für eine Modernisierung notwendig sind.

Von der anderen Seite, der journalistischen, kommt der Vorschlag von Matthias Karmasin, der nach einem Marktmodell des Journalismus und einem Moralmodell unterscheidet (vgl. Karmasin 1996, S. $226 \mathrm{ff}$.). Im Marktmodell lassen sich, so Karmasin, deutliche Defizite journalistischer Moral erkennen, während das Moralmodell eher zu Gewissenskonflikten führe.

Beiden Modellen gemeinsam ist, die Lösung im Ausgleich, im Gleichzeitigdenken von Medien und Journalismus, von ökonomischen Rationalitäten und publizistischer Autonomie, zu suchen. Ich dagegen plädiere dafür, die soziale Verantwortung nach derjenigen des Journalismus und derjenigen der Medien aufzuteilen. Dies hat verschiedene Vorteile:

- So können zum einen klare Referenzpunkte für das Maß an Verantwortung und Zuschreibungen für die Verantwortlichkeit von Akteuren verbindlicher fixiert werden.

- Zum anderen können dann auch die Rationalitäten der jeweiligen Bereiche angemessen berücksichtigt werden. Eine soziale Verantwortung der Medien erfordert die Integration verantwortlicher, ethischer Überlegungen in einen ökonomischen Rahmen. Eine soziale Verantwortung des Journalismus erfordert die Integration verantwortlicher, ethischer Überlegungen in den Rahmen des Journalismus.

Insgesamt plädiere ich für ein Ressourcenmodell der sozialen Verantwortung von Medien und ein Prozessmodell der professionellen Berichterstattung als soziale Verantwortung des Journalismus. Der Referenzpunkt für die soziale Verantwortung des Journalismus ist dann der Prozess der Aussagenentstehung. Dieser Prozess besteht aus verschiedenen Phasen, von der Themenfindung zur Recherche, vom Texten zum Gegenlesen. Verfehlungen können in den einzelnen Phasen dieses Prozesses festgemacht werden, bei der Nachrichtenselektion, bei der Recherche oder beim Gegencheck. Verfehlungen im Prozess der Aussagenentstehung können dann - entsprechend der 
Gliederung nach individueller, organisationaler oder professioneller Verantwortung - den Journalisten, den Redaktionen oder dem Berufsstand zugeschrieben werden und sind dementsprechend zu verantworten.

Der Referenzpunkt für die soziale Verantwortung der Medien ist das Maß an Ressourcen, das sie bereit sind, dem Journalismus zur Verfügung zu stellen. Diese Ressourcen können benannt, quantifiziert und bewertet werden, beispielsweise die personelle Besetzung der Redaktionen, die Höhe des Etats für Recherche und die Aufgabenbereiche respektive das Arbeitspensum der Journalisten. Im Abgleich mit den publizistischen Zielen der Redaktion kann durch einen Soll-/ Istvergleich festgestellt werden, ob ausreichend Ressourcen vorhanden sind. Publizistische Folgen einer Misswirtschaft oder einseitig profitorientierter Entscheidungen in Medienunternehmen können dann dem Medienmanagement zugeschrieben werden, dort liegt die Verantwortung für das medienwirtschaftliche Handeln.

Problematisch an solcherart Vorschlägen zur sozialen Verantwortung ist ihre Konkretheit. Werden, so die berechtigte Frage, die Medienunternehmen überhaupt einen Gedanken daran verschwenden? Und wollen die Medienunternehmen sich dementsprechend mit dem Journalismus auseinandersetzen? Wie soll es denn gelingen, Medien und Journalismus an den Verhandlungstisch zu bringen, damit das Ressourcen- und das Prozessmodell in der Praxis wirken können?

Nun, erstens kann mit einiger Beruhigung darauf verwiesen werden, dass Medien und Journalismus in Ko-Orientierung miteinander agieren, die von einer wechselseitigen Abhängigkeit geprägt ist: ohne journalistische Produktion keine Distribution, ohne Distribution keine Berichterstattung. Dies garantiert den Bestand der Ko-Orientierung. Zweitens haben die Vermutungen einige Berechtigung, die darauf aufmerksam machen, dass nicht wenige Medienunternehmen Erfolg mit journalistischer Qualität haben. Drittens schließlich ist auch der Medienbereich nicht so sehr bis in alle Winkel vermachtet, dass die konkreten Maßstäbe der Diskursethik keinerlei Beachtung finden würden (vgl. Brosda 2008; Funiok 1999). Die gegenseitige Anerkennung der Wahrheit des Widerspruchs von Erkenntnismaximierung und Profitmaximierung - und damit der Unterschiedlichkeit und Unterscheidbarkeit von Journalismus und Medien -, die (Rückkehr der) Wahrhaftigkeit als Grundlage der Kommunikation zwischen Journalismus und Medien und der Gerechtigkeit im Sinne einer Partizipation der journalistischen Organisationen an den Zukunftsplanungen und Investitionsentscheidungen, das sind auch Bestandteile einer sozialen Verantwortung des Journalismus wie der Medien. 
Dazu gäbe es dann im Übrigen auch schon einen ersten Prüfstein für diskursethische Verhandlungen zwischen Medien und Journalismus. Der Journalismus, so beschreibt die Wissenschaft seine Funktion, beobachtet die Gesellschaft und stellt ihr seine Beobachtungen wieder zur Verfügung (vgl. Löffelholz 2000). Der Journalismus betreibt dadurch (auch) Aufklärung. Es wäre überlegenswert und sinnvoll, würde der Joumalismus auch sich selbst einbeziehen in diese Aufklärung, durch den Medienjournalismus beispielsweise. Der Medienjournalismus, jener Teil des Journalismus, der über Medien berichtet, führt eine nur kümmerliche Existenz und versteht sich vor allem als Medienkritik. Würde er sich als Informant verstehen, der über die Bedingungen und Zwänge der Berichterstattung berichtet, dann wäre das Aufklärung im besten Sinne. Dann erfährt die Gesellschaft, wie Berichterstattung entsteht, die alle Gesellschaftsmitglieder benötigen, um an der politischen, wirtschaftlichen und kulturellen Entwicklung zu partizipieren. Ein Journalismus, der in der Gesellschaft agiert, muss sich selbst ebenso thematisieren wie die übrigen Bereiche der Gesellschaft. Auch das gehört zur sozialen Verantwortung des Journalismus.

Würde der Joumalismus dies ernst nehmen, wäre das auch der erste Prüfstein für die Medienunternehmen. Sie lesen nicht gern Reportagen aus den von ihnen beherrschten Redaktionen über die Bedingungen in ihrem Unternehmen, über schlechte Arbeitsbedingungen, Tarifausstiege, PR-Einfluss. Für diese Bedingungen aber sind sie verantwortlich. Statt in den Journalismus investieren sie lieber Kapital darin, neue Zielgruppen zu finden. Medienjournalismus zuzulassen und zu fördem wäre ein erster Prüfstein.

\section{Literatur}

Altmeppen, Klaus-Dieter (2006): Journalismus und Medien als Organisationen. Leistungen, Strukturen und Management. Wiesbaden.

Altmeppen, Klaus-Dieter /Ulrike Röttger/Günter Bentele (Hg.) (2004): Schwierige Verhältnisse. Interdependenzen zwischen Journalismus und PR. Wiesbaden.

Brosda, Carsten (2008): Diskursiver Journalismus. Journalistisches Handeln zwischen kommunikativer Vernunft und mediensystemischem Zwang. Wiesbaden.

Burkart, Roland/Walter Hömberg (2007): Elektronisch mediatisierte Gemeinschaftskommunikation. Eine Herausforderung für die kommunikationswissenschaftliche Modellbildung. In: dies. $(\mathrm{Hg})$ : Kommunikationstheorien. Ein Textbuch zur Einführung. 4. Aufl., Wien, S. 258-269.

Funiok, Rüdiger (Hg.) (1996): Grundfragen der Kommunikationsethik. Konstanz. 
Jarren, Otfried/Pascal Zwicky (2008): Es braucht kulturell eingebundenes Kapital. Die Finanzinvestoren und die Finanzierungsprobleme der Medien. In: NZZ Online vom 7.7.2008 (abgerufen am 7.7.2008).

Karmasin, Matthias (1996): Ethik als Gewinn. Zur ethischen Rekonstruktion der Ökonomie. Konzepte und Perspektiven von Wirtschaftsethik, Unternehmensethik, Führungsethik. Wien.

Löffelholz, Martin (Hg.) (2000): Theorien des Journalismus. Ein diskursives Handbuch, Wiesbaden.

Pimpl, Roland (2008): Korrektive gegen die Käuflichkeit. Mediaplaner begrüßen Doppelfunktion von Chefredaktion und Geschäftsführung/Genauere Titelüberprïfungen bei Sparplänen. In: Horizont, Nr. 28 vom 10.7.2008, S. 25. 